PROCEEDINGS OF THE

AMERICAN MATHEMATICAL SOCIETY

Volume 130, Number 3, Pages 753-758

S 0002-9939(01)06122-6

Article electronically published on August 28, 2001

\title{
A GENERALIZED KOLMOGOROV INEQUALITY FOR THE HILBERT TRANSFORM
}

\author{
MARK A. PINSKY
}

(Communicated by Christopher D. Sogge)

\begin{abstract}
If $f \in L^{1}\left(\mathbf{R}^{1} ;(1+|x|)^{-1} d x\right)$ we can define the Hilbert transform $H f$ almost everywhere (Lebesgue) and obtain an estimate for $\mu\{x:|H f(x)| \geq$ $\alpha$ \} where $\mu$ is a suitable finite measure. The classical Kolmogorov inequality for the Lebesgue measure of $\{x:|H f(x)| \geq \alpha\}$ is obtained by a scaling argument.
\end{abstract}

\section{INTRODUCTION}

The purpose of this note is to obtain an extended form of the Kolmogorov inequality for the Hilbert transform

$$
H f(x)=\frac{1}{\pi} \lim _{\epsilon \rightarrow 0} \int_{|y|>\epsilon} \frac{f(x-y)}{y} d y .
$$

If $f \in L^{p}(\mathbf{R})$ for some $p \in[1, \infty)$, the almost-everywhere existence of $H f$ can be obtained from an analysis of the conjugate Poisson kernel in the upper half-plane. The classical Kolmogorov inequality is the statement that

$$
\operatorname{Leb}\{x:|H f(x)| \geq \alpha\} \leq \frac{C}{\alpha} \int_{\mathbf{R}}|f(x)| d x, \quad \alpha>0, f \in L^{1}(\mathbf{R}) .
$$

However, the natural domain of definition of (1) is the Banach space

$$
B_{1}:=\left\{f: \int_{\mathbf{R}} \frac{|f(x)|}{1+|x|} d x<\infty\right\}
$$

which contains all of the Lebesgue spaces $L^{p}(\mathbf{R}), 1 \leq p<\infty$, where the operator $H$ has a well-established theory.

In order to study $H$ on $B_{1}$, we introduce the norms

$$
\|f\|_{B_{1}}:=\frac{1}{\pi} \int_{\mathbf{R}} \frac{|x f(x)|}{1+x^{2}} d x, \quad\|f\|_{B_{2}}:=\frac{1}{\pi} \int_{\mathbf{R}} \frac{|f(x)|}{1+x^{2}} d x .
$$

Define a weighted measure by

$$
\mu(A):=\frac{1}{\pi} \int_{A} \frac{d x}{1+x^{2}} .
$$

The main result is the following estimate.

Received by the editors March 14, 2000 and, in revised form, September 11, 2000.

2000 Mathematics Subject Classification. Primary 42A50; Secondary 44A15. 
Theorem. Suppose that $f \in B_{1}$. Then $H f(x)$ exists almost everywhere. If $f \geq 0$, then we have the estimate

$$
\mu\{x:|H f(x)| \geq \alpha\} \leq \frac{2}{\pi}\left(\frac{\|f\|_{B_{2}}}{\alpha-\|f\|_{B_{1}}}+\frac{\|f\|_{B_{2}}}{\alpha+\|f\|_{B_{1}}}\right), \quad \alpha>\|f\|_{B_{1}} .
$$

For any complex-valued $f \in B_{1}$, (6) holds with four terms on the right side, and where $\alpha$ is replaced by $\alpha / 4$.

In the final section we show how the classical Kolmogorov inequality (2) can be obtained from ([6). We also remark that precise constants for the classical Kolmogorov inequality (2) have been obtained by Davis [D]. Some recent generalizations have been discovered by Choi [C].

\section{Related properties of the Poisson Kernel}

In order to study the conjugate Poisson kernel, we first develop the necessary properties of the Poisson kernel operator, defined by

$$
P_{y} f(x):=\frac{1}{\pi} \int_{\mathbf{R}} \frac{y f(x-t)}{y^{2}+t^{2}} d t .
$$

This operator is defined on the Banach space

$$
B_{2}:=\left\{f:\|f\|_{B_{2}}:=\frac{1}{\pi} \int_{\mathbf{R}} \frac{|f(x)|}{1+x^{2}} d x<\infty\right\} .
$$

Clearly $L_{1}(\mathbf{R}) \subset B_{1} \subset B_{2}$. The Poisson kernel has the following properties.

Proposition 2.1. Suppose that $f \in B_{2}$. Then $\left\|P_{y} f\right\|_{B_{2}} \leq 2\|f\|_{B_{2}}$ for $0<y \leq 1$ and for any $f \in B_{2}, \lim _{y \rightarrow 0}\left\|P_{y} f-f\right\|_{B_{2}}=0$. Furthermore if $f \in B_{1}$, then $\lim _{y \rightarrow 0} P_{y} f(x)=f(x)$ for almost every $x \in \mathbf{R}$.

Proof. We have

$$
\begin{array}{rlr}
\left\|P_{y} f\right\|_{B_{2}} & \leq \frac{1}{\pi^{2}} \int_{\mathbf{R}}\left(\int_{\mathbf{R}} \frac{y|f(t)|}{y^{2}+(x-t)^{2}} d t\right) \frac{d x}{1+x^{2}} \\
& =\frac{1}{\pi^{2}} \int_{\mathbf{R}}|f(t)|\left(\int_{\mathbf{R}} \frac{y}{y^{2}+(x-t)^{2}} \frac{1}{1+x^{2}} d x\right) d t \\
& =\frac{1}{\pi} \int_{\mathbf{R}}|f(t)| \frac{1+y}{(1+y)^{2}+t^{2}} d t \\
& \leq \frac{1}{\pi} \int_{\mathbf{R}}|f(t)| \frac{2}{1+t^{2}} d t, \quad 0<y<1, \\
& =2\|f\|_{B_{2}}
\end{array}
$$

where we have used the semi-group property of the Poisson kernel in the form $P_{y} * P_{1}=P_{1+y}$. To prove the norm convergence, we first note that if $f=1_{[a, b]}$, then $\pi P_{y} f(x)=\arctan [(x-b) / y]-\arctan [(x-a) / y]$ which is bounded by 2 , and tends pointwise to $1_{[a, b]}$ except at the endpoints $x=a, b$. By the dominated convergence theorem, we have $\left\|P_{y} f-f\right\|_{B_{2}} \rightarrow 0$ when $y \rightarrow 0$. Similarly for a finite linear combination $f=\sum_{j=1}^{N} c_{j} 1_{\left[a_{j}, b_{j}\right]}$ we have $\left\|P_{y} f-f\right\|_{B_{2}} \rightarrow 0$. But these functions are dense in the space $B_{2}$, and we already have proved that the operator 
norms $\left\|P_{y}\right\|_{B_{2}}$ are uniformly bounded for $0<y \leq 1$, whence the result. To prove the almost-everywhere convergence, we write

$$
\begin{aligned}
P_{y} f(x)-f(x) & =\frac{1}{\pi} \int_{0}^{\infty}[f(x+t)+f(x-t)-2 f(x)] \frac{y}{t^{2}+y^{2}} d t \\
\left|P_{y} f(x)-f(x)\right| & \leq \int_{0}^{\infty} \frac{y}{t^{2}+y^{2}} d \Phi_{x}(t) d t \\
\Phi_{x}(t) & :=\frac{1}{\pi} \int_{0}^{t}|f(x+u)+f(x-u)-2 f(x)| d u .
\end{aligned}
$$

From Lebesgue's theorem, we have for almost every $x, \Phi_{x}(t) / t \rightarrow 0$ when $t \rightarrow 0$. On the other hand, $f \in B_{1}$ implies that $\Phi_{x}(t) \leq C t$ for all $t \geq 0$. Now we integrate by parts:

$$
\left|P_{y} f(x)-f(x)\right| \leq \int_{0}^{\infty} \frac{2 t y}{\left(t^{2}+y^{2}\right)^{2}} \Phi_{x}(t) d t
$$

where the estimate $\left|\Phi_{x}(t)\right| \leq C t$ allows one to discard the term at the limits. Setting $t=y z$ in the integration gives

$$
\left|P_{y} f(x)-f(x)\right| \leq \int_{0}^{\infty} \frac{2 z}{\left(1+z^{2}\right)^{2}} \frac{\Phi_{x}(z y)}{y} d z .
$$

But the integrand is bounded by an $L^{1}$ function and tends to zero pointwise when $y \rightarrow 0$, hence $P_{y} f(x) \rightarrow f(x)$ as required.

\section{Proof of the Theorem}

We follow the method of Carleson and Katznelson, as exposited in $\mathrm{Kz}$. Without loss of generality, we assume $f \geq 0$. Indeed, any complex-valued function can be written as $f=f_{1}-f_{2}+i\left(f_{3}-f_{4}\right)$ where $f_{j} \geq 0$. We begin with the conjugate Poisson kernel operator

$$
Q_{y} f(x)=\frac{1}{\pi} \int_{\mathbf{R}} \frac{t f(x-t)}{t^{2}+y^{2}} d t, \quad y>0, x \in \mathbf{R} .
$$

Clearly $\left|Q_{1} f(0)\right| \leq\|f\|_{B_{1}}$. Then

$$
P_{y} f(x)+i Q_{y} f(x)=\frac{i}{\pi} \int_{\mathbf{R}} \frac{f(t)}{x+i y-t} d t .
$$

For any $f \in B_{1}$ defines an analytic function in the upper half-plane $y>0$. The mapping

$$
(x, y) \mapsto \exp \left[-\left(P_{y} f(x)+i Q_{y} f(x)\right)\right]
$$

is a bounded analytic function in the upper half plane. By the Fatou theorem G], it possesses a.e. limits when $y \rightarrow 0$. But $P_{y} f(x)$ converges to a finite limit a.e. whenever $f \in B_{1} \subset B_{2}$. Hence we deduce the existence of the a.e. limit of $\exp \left[-i Q_{y} f(x)\right]$ when $y \rightarrow 0$. From this it follows that $Q_{y} f(x)$ can have only one accumulation point when $y \rightarrow 0$, hence the existence of $\tilde{H} f(x):=\lim _{y \rightarrow 0} Q_{y} f(x)$.

To prove (6), we consider the harmonic function $J_{\alpha}(w)$ defined for $\operatorname{Re}(w)>0$ as the harmonic measure of the two rays $\{w=i v, v \geq \alpha\}$ and $\{w=i v, v \leq-\alpha\}$. This is the harmonic function which takes the value 1 on these rays and takes the value zero on the segment $\{w=i v:-\alpha \leq v \leq \alpha\}$. Equivalently it can be obtained as the imaginary part of $(1 / \pi) \log [(w-i \alpha) /(w+i \alpha)]$ for a suitable branch of the 
logarithm. The set $\left\{w: J_{\alpha}(w) \geq 1 / 2\right\}$ is the exterior of the semi-circle described by $\{w: \operatorname{Re} w>0,|w|=\alpha\}$. On the strip $|\operatorname{Im}(w)|<\alpha$ we have

$$
J_{\alpha}(u+i v)=\frac{1}{\pi}\left[\arctan \left(\frac{u}{\alpha-v}\right)+\arctan \left(\frac{u}{\alpha+v}\right)\right], \quad u>0,|v|<\alpha .
$$

We now consider the harmonic function

$$
U_{\alpha}(x, y)=J_{\alpha}\left[P_{y} f(x)+i Q_{y} f(x)\right] .
$$

We first recall a basic fact from ( $\mathrm{G}$, p. 17, Lemma 3.4).

Lemma 3.1. If $U(x, y)$ is any bounded harmonic function in $y>0$, then for any $y_{1}, y_{2}>0$, we have

$$
U\left(x, y_{1}+y_{2}\right)=\frac{1}{\pi} \int_{\mathbf{R}} \frac{y_{2} U\left(t, y_{1}\right)}{(x-t)^{2}+y_{2}^{2}} d t .
$$

Applying (13) with $x=0, y_{2}=1, U=U_{\alpha}$, we have

$$
J_{\alpha}\left(P_{1+y} f(0)+i Q_{1+y} f(0)\right)=\frac{1}{\pi} \int_{\mathbf{R}} \frac{J_{\alpha}\left(P_{y} f(t)+i Q_{y} f(t)\right)}{1+t^{2}} d t .
$$

The right side of (14) is underestimated by

$$
\frac{1}{\pi} \int_{\mathbf{R}} \frac{J_{\alpha}\left(P_{y} f(t)+i Q_{y} f(t)\right)}{1+t^{2}} d t \geq \frac{1}{2 \pi} \int_{\left\{t:\left|Q_{y} f(t)\right| \geq \alpha\right\}} \frac{d t}{1+t^{2}} .
$$

Using the inequality $|\arctan (x)| \leq|x|$, applied to (12), we can overestimate the left side of (14) by writing

$$
J_{\alpha}\left(P_{1+y} f(0)+i Q_{1+y} f(0)\right) \leq \frac{1}{\pi}\left(\frac{P_{1+y} f(0)}{\alpha-\left|Q_{1+y} f(0)\right|}+\frac{P_{1+y} f(0)}{\alpha+\left|Q_{1+y} f(0)\right|}\right) .
$$

Therefore we have

$$
\begin{aligned}
\mu\left\{x:\left|Q_{y} f(x)\right| \geq \alpha\right\} & =\frac{1}{\pi} \int_{\left\{x:\left|Q_{y} f(x)\right| \geq \alpha\right.} \frac{d x}{1+x^{2}} \\
& \leq \frac{2}{\pi}\left(\frac{P_{1+y} f(0)}{\alpha-\left|Q_{1+y} f(0)\right|}+\frac{P_{1+y} f(0)}{\alpha+\left|Q_{1+y} f(0)\right|}\right) .
\end{aligned}
$$

In Section 4 (below) we prove that $H f(x)=\tilde{H} f(x):=\lim _{y \rightarrow 0} Q_{y} f(x)$ a.e., in particular we have convergence in measure. Now from (44), $\left|Q_{1} f(0)\right| \leq\|f\|_{B_{1}}$, $P_{1} f(0)=\|f\|_{B_{2}}$ and the right side of (15) is only increased when we replace $Q_{1} f(0)$ by its upper bound $\|f\|_{B_{1}}$. Hence

$$
\mu\{x:|H f(x)| \geq \alpha\} \leq \frac{2}{\pi}\left(\frac{\|f\|_{B_{2}}}{\alpha-\|f\|_{B_{1}}}+\frac{\|f\|_{B_{2}}}{\alpha+\|f\|_{B_{1}}}\right),
$$

which proves the result in case $f \geq 0$. In the general case, we write

$$
\mu\{x:|H f(x)| \geq \alpha\} \leq \sum_{j=1}^{4} \mu\left\{x:\left|H f_{j}(x)\right| \geq \alpha / 4\right\}
$$

and apply the result for non-negative functions to each of the terms on the right.

The upper bound assumes a more familiar form in case $f$ is even, as follows.

Corollary 3.1. Suppose that $0 \leq f \in B_{1}$ is even: $f(-x)=f(x), \forall x \in \mathbf{R}$. Then for any $\alpha>0$ we have

$$
\mu\{x:|H f(x)| \geq \alpha\} \leq \frac{4\|f\|_{B_{2}}}{\pi \alpha} .
$$


Proof. In this case we have $Q_{y} f(0)=0$ for all $y>0$. Thus the right side of (15) becomes $\frac{4}{\pi} P_{1+y} f(0) \rightarrow \frac{4\|f\|_{B_{2}}}{\pi}$ when $y \rightarrow 0$.

\section{Identification of $H$}

It remains to identify the Hilbert transform as defined in (11), with the boundary values of $Q_{y} f$, namely to show that for a.e. $x \in \mathbf{R}$,

$$
Q_{y} f(x)=\frac{1}{\pi} \int_{\mathbf{R}} \frac{t f(x-t)}{t^{2}+y^{2}} d t \rightarrow H f(x), \quad y \downarrow 0 .
$$

Proposition 4.1. Suppose that $f \in B_{1}$. Then

$$
\lim _{y \rightarrow 0}\left(\int_{\mathbf{R}} \frac{t f(x-t)}{t^{2}+y^{2}} d t-\int_{|t|>y} \frac{f(x-t)}{t} d t\right)=0
$$

for almost every $x \in \mathbf{R}$.

Proof. We write the above difference as $I_{1}+I_{2}$ where

$$
\begin{gathered}
I_{1}=\int_{|t|<y} \frac{t f(x-t)}{t^{2}+y^{2}} d t, \\
I_{2}=\int_{|t| \geq y}\left(\frac{t}{t^{2}+y^{2}}-\frac{1}{t}\right) f(x-t) d t .
\end{gathered}
$$

The function $t \rightarrow t /\left(t^{2}+y^{2}\right)$ is odd and increasing for $|t|<y$, so that we can write

$$
\begin{aligned}
I_{1} & =\int_{|t|<y} \frac{t}{t^{2}+y^{2}}[f(x-t)-f(x)] d t, \\
\left|I_{1}\right| & \leq \frac{1}{2 y} \int_{|t|<y}|f(x-t)-f(x)| d t \rightarrow 0
\end{aligned}
$$

at every Lebesgue point of $f$, especially almost everywhere.

To estimate $I_{2}$ we note that its kernel is odd, hence for any $\delta>0$ and $y<\delta$,

$$
\begin{aligned}
-I_{2} & =\int_{|t|>y} \frac{y^{2}}{t\left(t^{2}+y^{2}\right)}[f(x-t)-f(x)] d t, \\
\left|I_{2}\right| & \leq \int_{|t|>y} \frac{y^{2}}{|t|^{3}}|f(x-t)-f(x)| d t \\
& =y^{2} \int_{|t|>y} \frac{d F(t)}{|t|^{3}}
\end{aligned}
$$

where $F(t):=\int_{0}^{t}|f(x-s)-f(x)| d s$. Clearly $F(t) / t \rightarrow 0$ at every Lebesgue point when $t \rightarrow 0$, whereas $F(t) \leq C t$ when for all $t$. Therefore we can integrate-by-parts to obtain

$$
\int_{|t|>y} \frac{d F(t)}{|t|^{3}}=\frac{F(y)}{|y|^{3}}+3 \int_{|t|>y} \frac{F(t)}{t^{4}} d t
$$

The term at the limits is clearly $o\left(y^{-2}\right)$ when $y \rightarrow 0$. To analyse the new integral, write $F(t) / t=\eta(t), v=1 / t$ to obtain

$$
\int_{|t|>y} \frac{F(t)}{t^{4}} d t=\int_{0}^{1 / y} v \eta(1 / v) d v=o\left(y^{-2}\right), \quad y \rightarrow 0,
$$

which completes the proof that $I_{2} \rightarrow 0$ when $y \rightarrow 0$ for almost every $x \in \mathbf{R}$. 


\section{Retrieval of the Classical Kolmogorov inequality}

Our result (6) contains the classical Kolmogorov inequality (2) as a special case, when we introduce a scaling parameter $Y$. In detail, define

$$
\mu_{Y}(A)=\frac{1}{\pi} \int_{A} \frac{Y}{t^{2}+Y^{2}} d t .
$$

Then we have the following scaled replacement for (6) when $\alpha>\left|Q_{Y} f(0)\right|$ :

$$
\mu_{Y}\{x:|H f(x)| \geq \alpha\} \leq \frac{2}{\pi}\left(\frac{P_{Y} f(0)}{\alpha-Q_{Y} f(0)}+\frac{P_{Y} f(0)}{\alpha+Q_{Y} f(0)}\right) .
$$

Now multiply (18) by $Y$ and take $Y \rightarrow \infty$. For the left side, we note that for any Borel set of finite Lebesgue measure we have from the dominated convergence theorem

$$
\lim _{Y \rightarrow \infty} Y \mu_{Y}(A)=\frac{1}{\pi} \operatorname{Leb}(A) .
$$

For the right side, we see that when $Y \rightarrow \infty$, the dominated convergence theorem shows that for any $f \in L^{1}(\mathbf{R})$

$$
\lim _{Y \rightarrow \infty} Y P_{Y} f(0)=\frac{1}{\pi} \int_{\mathbf{R}} f(x) d x
$$

whereas

$$
\left|Q_{Y} f(0)\right| \leq\|f\|_{L^{1}(\mathbf{R})} \times \sup _{t \in \mathbf{R}} \frac{|t|}{t^{2}+Y^{2}} \rightarrow 0, \quad Y \rightarrow \infty .
$$

Hence when we multiply (18) by $Y$ and take $Y \rightarrow \infty$, we obtain the original form (2) of Kolmogorov's inequality.

\section{ACKNOWLEDGEMENT}

We would like to thank the referee for several useful comments, including a "real-variable" proof of (6).

\section{ADDED IN PROOF}

We have recently learned that a different approach to this problem has appeared in the book of Paul Koosis, The Logarithmic Integral. I, Cambridge Studies in Advanced Mathematics, vol. 12, Cambridge University Press, 1998, pp. 59-65.

\section{REFERENCES}

[C] C. Choi, A weak-type inequality for differentially subordinate harmonic functions, Transactions of the American Mathematical Society, 350(1998), 2687-2696. MR 99e:31006

[D] B. Davis, On the distribution of conjugate functions of non-negative measures, Duke Mathematical Journal, 40(1973), 695-700. MR 48:2649

[G] J. Garnett, Bounded Analytic Functions, Academic Press, 1981. MR 83g:30037

[Kz] Y. Katznelson, Introduction to Harmonic Analysis, Dover reprint, 1976. MR 54:10976

Department of Mathematics, Northwestern University, Evanston, Illinois 60208-2730

E-mail address: pinsky@math.nwu.edu 